\title{
STUDY OF THE CARRIER STATE FOR FIVE BRCA1/BRCA2 DELETERIOUS MUTATIONS IN BULGARIAN WOMEN WITH BREAST CANCER
}

\section{Katia S. Kovacheva, Zornica B. Kamburova, Savelina L. Popovska', Ivan N. Ivanov', Maria N. Simeonova, Petia N. Angelova}

Section of Medical Genetics, Medical University - Pleven ${ }^{1}$ Department of Pathology, Medical University - Pleven, Bulgaria

Corresponding Author:

Zornica B. Kamburova

Section of Medical Genetics

Medical University - Pleven

1, Kliment Ohridski str.

Pleven, 5800

Bulgaria

e-mail: zornicakamburova@gmail.com

Received: July 01, 2013

Revision received: December 09, 2013

Accepted: December 27, 2013

\section{Summary}

Genetic testing for BRCA1/2 mutation is a well recognized medical management tool. Identification of healthy carriers of such mutations allows effective risk reduction procedures to be performed. There is no data reported on the founder mutations in the Bulgarian population. To evaluate the contribution of genetic factors to breast cancer (BC), we investigated the carrier state of Bulgarian women with $\mathrm{BC}$ for five common (according to BIC database) deleterious BRCA1/2 mutations. The list of patients diagnosed with BC between January 2011 and April 2012 was obtained from the Cancer Registry of University Hospital, Pleven. Eighty-two women with BC were interviewed and a pedigree was constructed of each of them. The patients were classified into seven categories, according to personal, disease and family history. Based on the preliminary prepared selection criteria and the personal family history, we defined a target group of 33 Bulgarian women with BC. They were screened for five deleterious mutations: 5382insC in BRCA1 and 6174delT, 6079del4, 8138del5, 5946delCT in BRCA2, by DNA sequencing. The genetic analysis detected none of the tested mutations. Two polymorphic variants were found in BRCA2 gene: c.5744C $>\mathrm{T}$ (rs4987117, SNP database) in exon11E in one patient and c.7806-14T $>$ C (rs9534262, SNP database) in exon17 in 22 patients. In conclusion, without basic information on the founder mutations in the population, the genetic screening for the specific mutations in a small group of tested patients is ineffective.

Key words: BRCA1, BRCA2, Breast cancer, Bulgarian population, polymorphism

\section{Introduction}

A woman born in Bulgaria has a $7 \%$ average lifetime risk of being diagnosed with breast cancer (BC) [1]. This risk of $\mathrm{BC}$ is determined by both genetic and lifestyle factors. The first significant report concerning the genetics of $\mathrm{BC}$ was made by Broca in 1866 , who traced the cause of death of 38 members of his wife's family through five generations. He found an increased frequency of $\mathrm{BC}$ in female relatives of $\mathrm{BC}$ probands. Since then, familial clustering has been verified repeatedly in other studies [2].

Breast cancer is approximately twice as common 
— Kovacheva K., et al. Study of the carrier state for five brca1/brca2 deleterious mutations...

in women with an affected first degree relative; the risk increases with the number of affected relatives and is greater for women with relatives affected at a young age [3]. Many multiple-case families have been investigated to identify highrisk susceptibility genes, using linkage analysis to identify markers that cosegregate with the disease. This approach has led to the demonstration of linkage to chromosome $17 \mathrm{q}$ and to chromosome $13 \mathrm{q}$ in a subset of $\mathrm{BC}$ families. Subsequent positional cloning has resulted in identifying the BRCA1 and BRCA2 genes with mutations in families linked to these gene regions [4]. Germline BRCA1 or BRCA2 mutations account for $20-40 \%$ of $\mathrm{BC}$ that cluster in families and less than $5 \%$ of $\mathrm{BC}$ overall [5-9]. Hereditary $\mathrm{BC}$ is actually the inheritance of susceptibility to cancer rather than the cancer itself.

Many studies report the typical tumour characteristics of BRCA1-associated BC, such as lack of expression of estrogen receptor (ER), progesterone receptor (PR), and HER2 (defined as a triple-negative breast cancer - TNBC), and high histological grade. Nearly $75 \%$ of BRCA1associated $\mathrm{BC}$ is either TNBC, basal-like or both $[10,11]$. Although fewer data are available on BRCA2-associated BC, the phenotype appears to be partly similar to that of BRCA1 with respect to young age at diagnosis, increased risk of contralateral BC and high histological grade [12].

The Breast Cancer Information Core (BIC) database has recorded 1810 distinct germline BRCA1 mutations and 2037 for BRCA2 mutations [13]. Of these, 996 BRCA1 mutations (55\%) and 1094 BRCA2 mutations (54\%) have been reported just once. Mutations appear to be equally distributed across the coding sequences, with no obvious "mutation hot-spot" $[4,14,15]$.

Most of the reported disease-associated alleles of BRCA1/2 have been attributed to frameshift, nonsense or missense mutations, large rearrangements or splice alterations. They usually lead to truncation of BRCA1 or BRCA2 protein or affect aminoacids that are critical for the protein structure or function. However, a large number of sequence variants (particularly missense variations) cannot be distinguished with certainty as either disease-causing (deleterious) mutations or benign polymorphisms (clinically not significant). They are classified as variants of unknown clinical significance [16]. At present, no reliable functional assay exists to determine whether such a variant is likely to be deleterious. Only epidemiological evidence on the frequency of the variant in $\mathrm{BC}$ cases and controls, and on cosegregation of the variant with disease in families can be regarded as definitive. Unfortunately, there is no evidence for most of the variants $[4,14,15]$.

A healthy individual could inherit a germline mutation in one allele of BRCA1/BRCA2 while the other one is normal. However, a somatic mutation may arise in the second allele in one's lifetime. The inheritance pattern is of an autosomal dominant type, with a relatively high penetrance. Therefore, a female carrier of BRCA1/BRCA2 germline mutation could have more than $60 \%$ lifetime risk of being diagnosed with BC. By identifying a female carrier of such a germline mutation, appropriate prevention measures (mastectomy, hormonal therapy) can be used to decrease the risk [17].

Mutations found more often in certain ethnic groups are known as founder mutations. In the Bulgarian population (7 364570 people), an average of 3,400 new BC cases annually have been reported over the last 10 years (with a moderate tendency of increase), and 1,200 women die of $\mathrm{BC}$ each year $[1,18]$. There is no available data of prevalence and type of BRCA1/2 mutation for the Bulgarian population.

Without any reported data concerning the founder mutations in the Bulgarian population and in order to appreciate the contribution of genetic factors to $\mathrm{BC}$, we used the BIC data to select five of the most common deleterious BRCA1/2 mutations and investigate the carrier state of Bulgarian women with $\mathrm{BC}$ for those mutations.

\section{Material and Methods}

\section{Patients}

The list of the patients diagnosed with $\mathrm{BC}$ for the period between January 2011 and April 2012 and the information regarding their clinical history were provided by the Cancer Registry of University Hospital, Pleven.

Letters with information about the aim of the study and an invitation to participate were sent to all live female patients. The study was approved by the hospital's ethics and research committees.

The patients who responded to the invitation visited the medical genetics unit at the Medical University, Pleven. A questionnaire was prepared beforehand and then filled in by a genetic counselor during the interview with each woman. The questionnaire aimed to provide 
information about the patient's age, menarche and menstrual history, history of childbearing, breastfeeding, menopause, intake of oral contraceptives, hormonal therapy for menopause, diet, intake of alcohol and smoking, previous benign breast disease or some other type of cancer, and family history of $\mathrm{BC}$ or other cancer. A pedigree, including at least three generations of relatives, was constructed for each patient. A patient with $\mathrm{BC}$ was defined as a proband (individual from whom the family history was traced).

The probands were classified into seven categories, according to family and personal disease history: (1) probands with familial BC the occurrence of two or more first degree relatives in the pedigree including the proband; (2) probands with no family history but with early-onset $\mathrm{BC}(<50$ years); (3) probands with no family history but with bilateral BC; (4) probands with no family history but with both $\mathrm{BC}$ and ovarian cancer (OC); (5) probands with no family history but with TNBC; (6) probands without family history of BC but with family history of other associated cancers (ovarian, pancreatic, gastric and prostatic cancers); (7) probands without any of the criteria mentioned above.

Based on the literature data, we defined the criteria for selection of patients in our target group: patients who were more likely to carry germline mutation in BRCA1/2 gene. The target group included all women from groups 1, 2, 3, 4 and 5. They were referred for genetic testing after signing an informed consent form. The genetic screening for five of the most commonly reported [13] point mutations in BRCA1 and BRCA2 genes (5382insC in BRCA1 and 6174delT, 6079del4, 8138del5, 5946delCT in BRCA2) was performed at the Molecular Medicine Center, Medical University - Sofia.

\section{Screening for BRCA1 and BRCA2 germline mutation}

Genomic DNA was isolated from peripheral blood using standard protocols (CHEMAGEN ${ }^{\circledR}$ Magnetic Separation Station). To amplify exon 20 of BRCA1 and exons 11n and 17 of BRCA2 and exons boundaries, primer pairs were used. Primer selection was made from BIC database at http://research.nhgri.nih.gov/projects/bic/Memb er/brca1_mutation_database.shtml and http://research.nhgri.nih.gov/projects/bic/Memb er/brca2_mutation_database.shtml. Genomic DNA was amplified by the Polimerase Chain
Mutation analysis of PCR products was performed by direct DNA sequencing. Sequencing was done using Big Dye ${ }^{\circledR}$ Terminator kit v3.1, (Appilied Biosystems), according to manufacturer's instructions using ABI Prism $3130 \mathrm{xl}$ (Applied Biosystems) sequencer. The results were compared with the reference DNA sequences using SeqScape, Sequencing analysis and FinchTV software, and then reviewed manually. All mutations and sequence variants were named according to HGVS (nomenclature guidelines for cDNA sequence) and dbSNP .

\section{Results}

For the study period, there were 258 patients with $\mathrm{BC}$ recorded in the Cancer Registry of University Hospital, Pleven. Of all recorded patients, 5 were males and 13 dead females and they were initially excluded from the study. An invitation to take part in our study was sent to the rest of 240 live females of whom 77 (32\%) agreed to participate in the study at the Section of Medical genetics. Five more patients were referred from the Department of Surgical Oncology, University hospital, Pleven. The final total number of patients included in the study was 82 , with average age at $\mathrm{BC}$ diagnosis 57 years. All these patients were interviewed and pedigrees were constructed. On the basis of the selection criteria prepared beforehand and the family history, the patients were subdivided into the following groups: with familial BC -12 $(14.6 \%)$ women; with early age of onset $(<50$ years) - $19(23.2 \%)$; with bilateral BC - 3 women (3.7\%), with $\mathrm{BC}$ and $\mathrm{OC}$ - one (1.2\%) woman; with TNBC - $6(7.3 \%)$. The rest of the patients $41(50 \%)$ were without any specific characteristics of $\mathrm{BC}$ and were excluded from the study. The characteristics of all 82 probands, according to the selection criteria, are presented in detail on Table 1.

The final target group included 41 women who met the selection criteria. They were referred for genetic testing. As three patients with familial $\mathrm{BC}$ and five with early-onset $\mathrm{BC}$ refused to be tested for mutations, a total of 33 women were screened for mutation. Nine of them had family histories of BC, 14 women were with early-onset $\mathrm{BC}, 3$ with bilateral $\mathrm{BC}$, one of them had BC and OC. Six other patients were with TNBC. The genetic testing for one mutation in BRCA1 (5382insC) and four in BRCA2 (6174delT, 6079del4, 8138del5, 5946delCT) 
— Kovacheva K., et al. Study of the carrier state for five brca1/brca2 deleterious mutations...

gene was performed. None of these five deleterious mutations was detected in the patients screened. The DNA sequencing revealed two polymorphic variants in BRCA2 gene: c. 5744C $>$ T (rs4987117, SNP database) in exon11E in one patient and c.7806-14T $>\mathrm{C}$ (rs9534262, SNP database) in exon17 in 22 patients. Detailed information about the type and frequency of sequence variants detected is shown on Table 2.

Table 1. Characteristics of probands

\begin{tabular}{lll}
\hline Characteristics & $\begin{array}{l}\text { Number of probands } \\
\text { (total number=82) }\end{array}$ & $\mathbf{\%}$ \\
\hline Familial BC & 12 & 14.6 \\
& & \\
With one close relative with BC & 8 & 9.9 \\
With one close relative with BC & 1 & 1.2 \\
$\quad$ With one close relative with BC & 2 & 2.5 \\
\hline Early age of onset $(<50)$ & 19 & 23.1 \\
& & \\
$<40$ years & 5 & 6.1 \\
$40-50$ years & 14 & 17 \\
\hline Bilateral BC & 3 & 3.7 \\
\hline BC and Ovarian cancer & 1 & 1.2 \\
\hline Triple-negative BC & 6 & 7.3 \\
\hline With other cancer in family & 15 & 18.3 \\
\hline Without any high risk features & 26 & 31.7 \\
\hline
\end{tabular}

Table 2. Allele frequencies of the polymorphic variants: Comparison of frequency in the European populations published in HAPMAP and the frequency found in our study

\begin{tabular}{lllllll}
\hline SNP & \multicolumn{2}{l}{ SNP frequency (according to CSHL- } & \multicolumn{2}{l}{ SNP frequency in probands of our study } \\
& HAPMAP:HapMap-CEU) & & & & \\
\hline Genotype & Wild-type & Heterozygous & Homozygous & Wild-type & Heterozygous & Homozygous \\
\hline rs9534262 & TT-26.5\% & TC-39.8\% & CC-33.6\% & $\begin{array}{l}\text { TT-33\% } \\
(11 / 33)\end{array}$ & $\begin{array}{l}\text { TC-36.6\% } \\
(12 / 33)\end{array}$ & $\begin{array}{l}\text { CC-30.4\% } \\
(10 / 33)\end{array}$ \\
\hline rs4987117 & CC-93.6\% & CT-6.4\% & TT-1.1\% & CC-96.9\% & CT-3.1\% & TT-0\% \\
& & & & $(32 / 33)$ & $(1 / 33)$ & $(0 / 33)$ \\
\hline
\end{tabular}

\section{Discussion}

It is known that female $\mathrm{BC}$ incidence is strongly related to age, with the highest overall incidence rates in older women, supporting a link with hormonal status. In the UK between 2008 and 2010 , an average of $20 \%$ of women with BC was diagnosed before the age of 50 [19]. In our study, the average age at diagnosis of $\mathrm{BC}$ was 57 years and about $23 \%$ of the patients were younger than 50 years.

The average rate of familial $\mathrm{BC}$ estimated to be $10-20 \%$ of all $\mathrm{BC}$ cases [20]. We detected similar incidence of familial BC $-14.7 \%$ and, in most of these cases $(66.7 \%)$, the proband had only one close relative with BC.
According to current estimates, TNBC accounts for an average of $10-17 \%$ of BC, depending on the thresholds used to define ER and PR positivity and HER2 over expression [21]. In different studies and patient populations, TNBC may range from $6 \%$ to $28 \%$ of $\mathrm{BC}$ [22$25]$. The incidence of TNBC that we found in our target group was $7.3 \%$. The relatively low incidence established could be explained with the small number of the patients studied and the strict criteria used to define a negative steroid receptor status.

Bilateral $\mathrm{BC}$ is rare and the reported incidence varies from $1 \%$ to $14 \%$ [26-28]. This type of BC is more frequent among women aged under 50 at first diagnosis [29]. Young age at primary BC 
diagnosis is associated with an increased susceptibility to bilateral $\mathrm{BC}$, mainly due to the likelihood of living long enough to develop contralateral BC. In our study, three (3.7\%) of all $82 \mathrm{BC}$ patients had bilateral $\mathrm{BC}$ and two of them $(66 \%)$ were with early ( $<50$ years of age) onset of the disease.

An unaffected woman who is a carrier of mutation in BRCA1/2 genes has between 56\% and $87 \%$ lifetime risk to develop BC by age 70 . The genetic testing for BRCA1/2 mutation is a well recognized and useful medical management tool. The identification of healthy carriers of such mutations allows effective risk reduction procedures (prophylactic mastectomy) and screening measures (breast MRI) to be performed [17].

In our study, the genetic testing of five deleterious mutations in BRCA1 and BRCA2 gene, preliminary selected based on the BIC data, did not reveal any of these specific defects in the investigated Bulgarian population of women with BC. It might be due to: (1) The small number of the tested patients in our final target group (with expected low prevalence of BRCA $1 / 2$ mutations - about $5 \%$ of all BC cases); (2) BRCA1 and BRCA2 genes together consist of approximately 20000 nucleotides and the mutations are spread in the entire sequences with no obvious "mutation hot-spot" [15]. Obviously, genetic screening for specific mutations, without basic information concerning the founder mutations in the population, is ineffective; (3) Although the most common BRCA mutations are point mutations and usually detected by sequencing analysis, the large genomic rearrangements comprise a significant component of identifiable mutations in BRCA1 and BRCA2 genes. These rearrangements cannot not be identified using a PCR-based method. An increase in the rate of mutation detection could be achieved by including Multiplex ligation-dependent probe application (MLPA) and highly sensitive DNA-based quantitative techniques of mutation screening [30].

\section{References}

1. Bulgarian national cancer registry. Cancer incidence in Bulgaria - data for 2010. [cited 2013 Nov 2]. Available from:

http://www.sbaloncology.bg/en/ncr.html
Concerning the polymorphisms detected in our study, one of our patients was with heterozygous state for polymorphism c. $5744 \mathrm{C}>\mathrm{T}$ in BRCA2 gene. This woman was diagnosed with TNBC at the age of 39 years. This polymorphism is a missense mutation that causes the change of amino acid sequence in BRCA2 from threonine to methionine at codon 1915. It is one of the missense BRCA2 mutations with a relatively high penetrance and unknown clinical significance. Studies carried out on the Polish population have concluded that the heterozygote state $(\mathrm{CT})$ for this mutation is associated with early onset $\mathrm{BC}$ and decreases the risk for the disease (above 40 years of age); and that the homozygote state (TT) is associated with a later age of cancer (above 40 years of age) [31,32].

The second polymorphic variant c.7806$14 \mathrm{~T}>\mathrm{C}$ in BRCA2 was found in 22 of our patients. This is an intronic sequence variant with unknown clinical significance. We did not find any published data on the association between this variant and increased risk of $\mathrm{BC}$ for any population.

\section{Conclusion}

The incidence rate of familial BC (about 15\%) in the Bulgarian population is similar to that in other European populations. Genetic screening for the specific point mutations in a small group of tested patients, without basic information concerning the founder mutations in the population is ineffective. In a future investigation, in order to increase the rate of mutation detection, genetic screening should combine methods for identification of both point mutations and large genomic rearrangements.

\section{Acknowledgement}

This study was supported by project № 9/2012 of Medical University of Pleven, Bulgaria.

2. Broca P, Traite' des tumeurs. Paris: P. Asselin; 1866.

3. Collaborative Group on Hormonal Factors in Breast Cancer. Familial breast cancer: collaborative reanalysis of individual data from 52 epidemiological studies including 58209 women with breast cancer and 101986 women without the 
— Kovacheva K., et al. Study of the carrier state for five brca1/brca2 deleterious mutations...

disease. Lancet 2001;358(9291):1389-99.

4. Thompson D, Easton D. The genetic epidemiology of breast cancer genes. J Mammary Gland Biol Neoplasia. 2004;9(3):221-36.

5. American College of Obstetricians and Gynecologists; ACOG Committee on Practice Bulletins-Gynecology; ACOG Committee on Genetics; Society of Gynecologic Oncologists. ACOG Practice Bulletin No. 103: Hereditary breast and ovarian cancer syndrome. Obstet Gynecol 2009;113(4):957-66.

6. National Cancer Institute [Internet]. Genetic testing for hereditary cancer syndromes. [updated 2013 Oct 4; cited 2013 Nov 2]. Available from: http://www.cancer.gov/cancertopics/factsheet/Ris $\mathrm{k} /$ genetic-testing

7. Hughes KS, Ravnikar V. Risk assessment and management of women with BRCA mutations. A Supplement to The Female Patient. 2009, Nov.

8. National Cancer Center Network. Clinical Practice Guidelines in Oncology: Genetic/Familial High-Risk Assessment: Breast and Ovarian. [cited 2013 Nov 2]. Available at: www.nccn.org/professionals/physician_gls/ PDF/genetics_screening.pdf.

9. Wooster R, Weber BL. Breast and ovarian cancer. N Engl J Med. 2003;48(23):2339-47.

10. Lakhani SR, Jacquemier J, Sloan JP, Gusterson BA, Anderson TJ, van de Vijver MJ, et al. Multifactorial analysis of differences between sporadic breast cancers and cancers involving BRCA1 and BRCA2 mutations. J Natl Cancer Inst. 1998;90(15):1138-45.

11. Narod SA, Foulkes WD. BRCA1 and BRCA2: 1994 and beyond. Nat Rev Cancer. 2004;4(9): 665-76.

12. Agnarsson BA, Jonasson JG, Björnsdottir IB, Barkardottir RB, Egilsson V, Sigurdsson $H$. Inherited BRCA2 mutation associated with high grade breast cancer. Breast Cancer Res Treat. 1998;47(2):121-7.

13. National Human Genome research Institute [Internet]. Breast cancer information core Breast cancer. [cited 2013 Nov 2]. Available from: http://research.nhgri.nih.gov/projects/bic/

14. Peto J, Collins N, Barfoot R, Seal S, Warren W, Rahman N, et al. Prevalence of BRCA1 and BRCA2 gene mutations in patients with earlyonset breast cancer. J Natl Cancer Inst. 1999;91(11):943-9.

15. Serova OM, Mazoyer S, Puget N, Dubois V, Tonin $\mathrm{P}$, Shugart YY, et al. Mutations in BRCA1 and BRCA2 in breast cancer families: are there more breast cancer-susceptibility genes? Am J Hum Genet. 1997;60(3):486-95.

16. Levenat S, Musani V, Cvok ML, Susac I, Sabol M, Ozretic P, et al. Three novel BRCA1/BRCA2 mutations in breast/ovarian cancer families in Croatia. Gene. 2012;498(2):169-76.

17. Tucker JM, Rizk B. Hereditary female cancers:
Breast, ovarian, and endometrial. Middle East Fertil Soc J. 2011;16(4):241-7.

18. National statistic institute [Internet]. Mortality by causes, sex and age. [cited 2013 Nov 2]. Available from: http://www.nsi.bg/otrasal.php?otr=22

19. Cancer Research UK [Internet]. Breast cancer. Breast cancer incidence statistics. [updated 2012 Nov 7; cited 2013 Nov 2].Available from: http://www.cancerresearchuk.org/cancerinfo/cancerstats/types/breast/incidence/uk-breastcancer-incidence-statistics

20. Evans DGR, Fentiman IS, McPherson K, Asbury D, Ponder BAJ, Howell A. Familial breast cancer. BMJ. 1994;308:183-7.

21. Reis-Filho JS, Tutt AN. Triple negative tumours: a critical review. Histopathology. 2008;52(1):10818.

22. Haffty BG., Yang Q, Reiss M, Kearney T, Higgins $\mathrm{SA}$, Weidhaas $\mathrm{J}$, et al. Locoregional relapse and distant metastasis in conservatively managed triple negative early-stage breast cancer. J Clin Oncol. 2006;24(36):5652-7.

23. Rakha EA, El-Sayed ME, Green AR, Lee AH, Robertson JF, Ellis IO. Prognostic markers in triple-negative breast cancer. Cancer. 2007;109(1):25-32.

24. Dent R, Trudeau M, Pritchard KI, Hanna WM, Kahn HK, Sawka CA, et al. Triple-negative breast cancer: clinical features and patterns of recurrence. Clin Cancer Res. 2007;13(15 Pt 1): 4429-34.

25. Kwan ML, Kushi LH, Weltzien E, Maring B, Kutner SE, Fulton RS, et al. Epidemiology of breast cancer subtypes in two prospective cohort studies of breast cancer survivors. Breast Cancer Res. 2009;11(3):R31.

26. Leis HP Jr. Managing the remaining breast. Cancer. 1980;46( Suppl 4):1026-30.

27. Adami HO, Bergstrom R, Hansen J. Age at first primary as a determinant of the incidence of bilateral breast cancer. Cancer. 1985;55(3):643-7.

28. Engin K. Prognostic factors in bilateral breast cancer. Neoplasma. 1994;41(6):353-7.

29. Rogozińska-Szczepka J, Utracka-Hutka B, Grzybowska E, Maka B, Nowicka E, SmokRagankiewicz A, et al; BRCA1 and BRCA2 mutations as prognostic factors in bilateral breast cancer patients. Ann Oncol. 2004;15(9): 1373-6.

30. Palma MD, Domchek SM, Stopfer J, Erlichman J, Siegfried JD, Tigges-Cardwell J, et al. The relative contribution of point mutations and genomic rearrangements in BRCA1 and BRCA2 in highrisk breast cancer families. Cancer res. 2008;68(17):7006-14.

31. Gorski B, Narod S, Lubinski J. A common missense variant in BRCA2 predispose to early onset breast cancer. Breast Cancer Res. 2005;7(6):R1023-7.

32. Sliwinski T, Krupa R, Majsterek I, Rykala J, Kolacinska A, Morawiec Z, et al. Polymorphisms of the BRCA2 and RAD51 genes in breast cancer. Breast Cancer Res Treat 2005;94(2):105-9. 